\title{
Asymptotic Behaviour of Neutrino Fields in Curved Space-time ${ }^{\star}$
}

\author{
J. AUDRETSCH \\ Fachbereich Physik der Universität Konstanz
}

Received February 15, 1971

\begin{abstract}
It is shown that, if a solution of the Weyl equation in some Riemann space is asymptotically smooth and describes a neutrino field with causal behaviour, than the order of the radiation term in the corresponding energy-momentum tensor predominates asymptotically the orders of the other terms.
\end{abstract}

\section{Introduction}

Usually, in General Relativity matter is described phenomenologically or by singularities. In contrast to this, a rigorous microphysical fieldtheory of matter must be based on a generalization of relativistic quantum mechanics, which first establishes matter in curved space-time on the same theoretical level as electrodynamics. In doing so, direct analogies to the well known electrodynamic fields are to be expected in case of the quantum mechanical zero rest-mass field of neutrinos described by the generally covariant Weyl theory (Section 2). Recently some topics of this theory of the gravitational interaction of neutrinos were discussed by Griffiths and Newing [1-3], Wainwright [4], Trim [5], Audretsch and Graf [6]. The direct physical significance of the theory results from the important role which neutrinos play, interacting with strong gravitational fields in some processes of stellar evolution and cosmology.

Continuing the investigations cited above, our main purpose in this paper is to study the asymptotic behaviour of the neutrino energymomentum tensor $T^{\alpha \beta}$ in curved space-time with the sole aid of generally covariant assumptions about the nature of the Weyl field. In particular, we want to show that these Weyl fields (in analogy to certain gravitational and electromagnetic fields) behave asymptotically like neutrino radiation.

For gravitational fields, radiation is defined by the Petrov type $N$ of the conformal tensor $C_{\alpha \beta \gamma \delta}$ (comp. [7]), whereas for the remaining physical fields, radiation is characterised by the Plebanski type $[4 \mathrm{~N}]_{2}$ (comp. [8]) of the energy-momentum tensor $T^{\alpha \beta}$. Especially in the case

\footnotetext{
* Dedicated to Prof. Dr. Helmut Hönl on the occasion of his retirement.
} 
of the neutrino radiation field, $T^{\alpha \beta}$ is composed out of the null 4-current $j^{\alpha}$ of the probability density according to ${ }^{1} T^{\alpha \beta} \sim j^{\alpha} j^{\beta}$. It will be shown that the assumptions made below lead to a geodesic $j^{\alpha}$-congruence. Therefore we can parametrize this congruence affinely, introducing the corresponding tangent vector $k^{\alpha}\left(k^{\alpha} \sim j^{\alpha}\right)$ and complete $k^{\alpha}(x)$ to a null frame field $k^{\prime \alpha}, n^{\prime \alpha}, m^{\prime \alpha}, \bar{m}^{\prime \alpha}$, which is parallelly propagated along the $j^{\alpha}$-streamlines. The decomposition of $T^{\alpha \beta}$ with respect to this null frame field becomes ${ }^{2}$

$$
\begin{aligned}
& T^{\alpha \beta}=\tau_{1} k^{\prime \alpha} k^{\prime \beta}+\tau_{2} k^{\prime(\alpha} n^{, \beta)}+\tau_{3} n^{\prime \alpha} n^{\prime \beta}+\tau_{4} m^{\prime(\alpha} \bar{m}^{, \beta)} \\
& +\operatorname{Re}\left(\tau_{5} k^{(\alpha} m^{\prime \beta)}+\tau_{6} n^{\prime(\alpha} m^{, \beta)}+\tau_{7} m^{\prime \alpha} m^{, \beta}\right) .
\end{aligned}
$$

Our problem then is to prove that moving along the $j^{\alpha}$-lines, $\tau_{1}$ is asymptotically of greater order in the affine parameter than the other $\tau_{i}$.

A possible starting point of our calculations could be the work of Penrose [9], who pointed out that under certain conditions the zero rest-mass fields itselves show an asymptotic peeling-off behaviour of the principal null directions. For the conformal tensor $C_{\alpha \beta \gamma \delta}$ and the Maxwell energy-momentum tensor (which is composed algebraically out of the field tensor ${ }^{3}$ ) this immediately implies the asymptotic passage to the Petrov and Plebanski type $N$ respectively ${ }^{4}$. However, for the Weyl field $\varphi_{A}$, being a spinor of rank one, the peeling-off property reduces to a statement about the order of the field in infinity. On the other hand, all $\tau_{n}$ in (1.1) are composed out of contractions of derivatives of $\varphi_{A}$ with vectors of the null frame (comp. (2.5)). Hence, in this case, no direct conclusion about the behaviour of $T^{\alpha \beta}$ can be drawn from the peeling-off property or from the asymptotic order of the field.

Therefore, instead of refering to the results of Penrose, we use the concept of asymptotic smoothness of $\varphi_{A}$ (Section 5). Further assumptions concerning the metric are not necessary! However, it is a general property of non-second quantised quantum mechanics that additional conditions are necessary to exclude un-physical fields (comp. the idea of the "Dirac sea"). In this respect we restrict ourselves to the very general local condition of causality leading to an energy property (Section 3), which implies a very simple form of the decomposition (1.1) of $T^{\alpha \beta}$ (Section 4). The asymptotic smoothness then immediately allows the intended statement about the asymptotic behaviour ot the components of $T^{\alpha \beta}$ (Section 6).

\footnotetext{
$1 \sim$ is the sign of proportionality.

${ }^{2}:=$ is the sign of definition. $M_{(\alpha \beta)}:=\frac{1}{2}\left(M_{\alpha \beta}+M_{\beta \alpha}\right), M_{[\alpha \beta]}:=\frac{1}{2}\left(M_{\alpha \beta}-M_{\beta \alpha}\right)$. (same rule for spinor indices). Re and Im denote the real and imaginary part of a complex quantity.

${ }^{3} F^{\alpha \beta} \leftrightarrow \varepsilon_{A B} \Phi_{\dot{W} \dot{X}}+\varepsilon_{\dot{W} \dot{X}} \Phi_{A B}, T^{\alpha \beta} \leftrightarrow \Phi_{A B} \Phi_{\dot{W} \dot{X}}$.

${ }^{4}$ Using other assumptions, this is deduced previously for the conformal tensor by Bondi et al. [10], Sachs [11], Newman and Penrose [12]. For the coupled EinsteinMaxwell field it is shown by Kozarzewski [13], compare also Jordan et al. [14].
} 


\section{Interaction of Neutrinos with the Gravitational Field}

In the framework of General Relativity the gravitational behaviour of neutrinos is determined by the gravitational field equation, for instance the Einstein equation

$$
R^{\alpha \beta}-\frac{1}{2} R g^{\alpha \beta}=-T^{\alpha \beta},
$$

and by the generally covariant Weyl equation in the 4-dim. Riemann space. In the 2 -spinor calculus ${ }^{5}$, the latter is given by (comp. $\left.[15,16]\right)$ :

$$
\sigma^{\alpha A \dot{B}} \varphi_{A \| \alpha}=0 \quad \text { or } \quad \varphi^{A} \| A \dot{B}=0 .
$$

In the following, not only the metric $g_{\alpha \beta}$ but also the Weyl field $\varphi_{A}$ will be treated as unquantised (i.e. classical) fields according to quantum mechanics before the second quantisation. This is justified because, with regard to our problem, we are not interested in the weak interaction or in the creation and annihilation of neutrinos by gravitons.

The physical behaviour of the neutrino field is described by the null 4-current $j^{\alpha}$ of the probability density

$$
j^{\alpha} \leftrightarrow \varphi_{A} \varphi_{\dot{B}},
$$

with

$$
j^{\varepsilon}{ }_{\| \varepsilon}=0, \quad j^{\varepsilon} j_{\varepsilon}=0,
$$

and the energy-momentum tensor $T^{\alpha \beta}$ (an irrelevant factor is neglected)

with

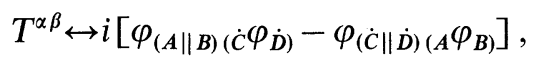

$$
T^{\alpha \beta}{ }_{\| \beta}=0, \quad T^{[\alpha \beta]}=0, \quad T_{\varepsilon}^{\varepsilon}=0, \quad T_{\alpha \beta} j^{\alpha} j^{\beta}=0 . \quad(2.6 \mathrm{a}-\mathrm{d})
$$

The analogue of $j^{\alpha}$ is the energy flow 4-vector $p^{\alpha}$. It depends on the 4-velocity $u^{\alpha}\left(u^{\varepsilon} u_{\varepsilon}=+1\right)$ of the measuring observer:

$$
p^{\alpha}(u):=T^{\alpha \beta} u_{\beta} .
$$

${ }^{5}$ Signature of $g_{\alpha \beta}:(---+)$. Partial and covariant derivatives are denoted ${ }_{\mid \alpha}$ and ${ }_{\| \alpha}$ respectively (same rule for spinor indices). The generalised Pauli matrices $\sigma^{\alpha A \dot{B}}$ are implicitly defined by

$$
2 \sigma_{(\alpha}{ }^{\dot{A} B} \sigma_{\beta) \dot{A} C}:=g_{\alpha \beta} \delta_{C}^{B}, \quad\left(\sigma^{\alpha \dot{A} B}\right)_{\| \varepsilon}=0 .
$$

The spinor equivalent $W_{R \dot{X}}^{A \dot{F} B \dot{G} \cdots}$ of the tensor $W_{\varrho}^{\alpha \beta \cdots}$ is given by

$$
W_{R \dot{X}}^{A \dot{B} B \dot{G} \cdots}:=\sigma_{R \dot{X}}{ }^{e} \cdots \sigma_{\alpha}^{A \dot{F}} \sigma_{\beta}^{B \dot{G}} \cdots W_{Q}^{\alpha \beta \cdots},
$$

with the inversion

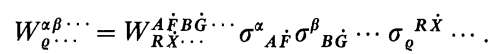

We abbreviate $W_{R \dot{X}}^{A \dot{F} B \dot{G}} \leftrightarrow W_{\varrho}^{\alpha \beta}$. Further details concerning the 2-spinor calculus in Riemann space can be found for instance in $[15,17-19]$. 
Then the probability density $W(u)$ and the energy density $E(u)$, as measured by the observer $u^{\alpha}$, are given by

$$
W(u):=j^{\varepsilon} u_{\varepsilon}, \quad E(u):=p^{\varepsilon} u_{\varepsilon} .
$$

Similarely $p^{\varepsilon} N_{\varepsilon}$ is the energy flux per unit time across a unit 2-surface with the normal $N^{\alpha}\left(N^{\varepsilon} N_{\varepsilon}=-1\right)$ which is situated in the observer's instantaneous rest space $\left(N^{\varepsilon} u_{\varepsilon}=0\right) . j^{\varepsilon} N_{\varepsilon}$ represents the corresponding particle flux.

These interpretations are generally valid for any field. For the Weyl field (as for all other quantum mechanical fields), they can be illustrated additionally regarding the structure (2.3) and (2.5) of $j^{\alpha}$ and $T^{\alpha \beta}$. In order to do so, we reintroduce with the aid of the tetrad-notation the standard Pauli matrices (comp. [16]), taking $u^{\alpha}$ as the time-like tetrad vector. We then obtain for example in (2.8b) the 3-density of the quantum mechanical expectation value of the energy, as built with the Hamilton operator in the well known manner. In this way, the probability interpretation of quantum mechanics enters the general theory of measurement in curved space-time.

\section{Causality}

It is well known, that the Weyl as well as the Dirac equation admit solutions with negative energy. Therefore, to exclude negative energies, in the first quantised Dirac theory in flat space-time the concept of the "Dirac sea" is used, which represents a global (!) restriction (concerning the integral value of the energy on a space-like hypersurface). On the contrary we will restrict ourselves to an energy condition, which is only local and means that the presence (that is the non-vanishing) and the sign of the energy $E(u)$ is independent of the oberver's velocity $u^{\alpha}$. In order to explain that this represents a selfevident physical restriction, we will derive it from the local causality condition, which is at the very basis of relativity theory. (The following is valid for every energymomentum tensor $T^{\alpha \beta}$.)

Causality. The maximum speed of any energy flow is the velocity of light. In other words, for any observer $u^{\alpha}\left(u^{\varepsilon} u_{\varepsilon}=+1, u^{\alpha}\right.$ future-pointing) we have, comp. (2.7),

$$
p^{\varepsilon}(u) p_{\varepsilon}(u) \geqq 0 .
$$

By (3.1) it is not explicitly excluded that $p^{\alpha}(u)$ vanishes for certain or all observers and also that for the same $T^{\alpha \beta}$ some $p^{\alpha}(u)$ are futurepointing and others past-pointing. The contents of the following theorem is that causality implicitly prevents both, which among other things 
means, that causality includes Wainwright's weak energy condition $E_{2}[4]$ (comp. the energy condition in [3]).

Energy property: If at a point $P$

a) $T^{\alpha \beta} \neq 0$ and

$\beta) T^{\alpha \beta}$ satisfies the causality condition (3.1), then one and only one of the following relations is valid for all observers $u^{\alpha}$ in $P$ :

$$
T^{\alpha \beta} u_{\alpha} u_{\beta}>0 \quad \text { or } \quad T^{\alpha \beta} u_{\alpha} u_{\beta}<0 .
$$

Proof. There are only the following two possibilities:

A) Every $p^{\alpha}(u)$ is equal to zero or for all $u^{\alpha}$ parallel to one certain direction $A^{\alpha}\left(A^{\varepsilon} A_{\varepsilon} \geqq 0\right)$.

B) There exist at least two non-vanishing $p^{\alpha}(u)$ which are not parallel to each other, say $\tilde{p}^{\alpha}:=T^{\alpha \beta} \tilde{u}_{\beta}$ and $\hat{p}^{\alpha}:=T^{\alpha \beta} \hat{u}_{\beta}$.

Case A: $T^{\alpha \beta}$ satisfies

$$
T^{\alpha \beta} u_{\alpha}=c(u) A^{\beta}
$$

for all $u^{\alpha}(c(u)$ not necessarily $\neq 0)$. Contraction with $B^{\beta}$ defined by $A_{\varepsilon} B^{\varepsilon}:=1$ gives

$$
c(u)=T^{\alpha \beta} u_{\alpha} B_{\beta},
$$

so that (3.3) may be rewritten as

$$
\left(T^{\alpha \beta}-T^{\alpha \varepsilon} B_{\varepsilon} A^{\beta}\right) u_{\alpha}=0 .
$$

Because of (3.5) being valid for all $u^{\alpha}$ we have

$$
T^{\alpha \beta}=T^{\alpha \varepsilon} B_{\varepsilon} A^{\beta} \quad \text { and } \quad 0=T^{[\alpha \beta]}=B_{\varepsilon} T^{\varepsilon[\alpha} A^{\beta]}, \quad(3.6 \mathrm{a}, \mathrm{b})
$$

and therefore

$$
A^{\alpha} \sim B_{\varepsilon} T^{\varepsilon \alpha} \quad \text { and } \quad T^{\alpha \beta} \sim A^{\alpha} A^{\beta} .
$$

Finally, (3.2) follows from (3.7b) together with $\alpha$ ).

Case B: We perform the proof by contradiction and assume, with $\alpha), \beta)$ and B) satisfied, that there may exist an $v^{*}$ with

$$
\stackrel{*}{p}^{\alpha}:=T^{\alpha \beta}{\stackrel{*}{u_{\beta}}}=0
$$

Using $\bar{u}^{\alpha}$ defined by

$$
\bar{u}^{\alpha}:=\stackrel{*}{u} \alpha^{\alpha}-\left(2{ }^{*} \varepsilon \tilde{u}_{\varepsilon}\right) \tilde{u}^{\alpha}, \quad \bar{u}^{\varepsilon} \bar{u}_{\varepsilon}=1,
$$

we form $\bar{p}^{\alpha}:=T^{\alpha \beta} \bar{u}_{\beta}$, which becomes according to (3.8) and (3.9a)

$$
\bar{p}^{\alpha}=-\left(2 *^{*} \tilde{u}_{\varepsilon}\right) \tilde{p}^{\alpha} \neq 0, \quad \tilde{u}^{\varepsilon} \tilde{u}_{\varepsilon}>0 .
$$

Thus $\bar{p}^{\alpha}$ is antiparallel to $\tilde{p}^{\alpha}$. Therefore and because of B) we can choose under $\tilde{p}^{\alpha}, \hat{p}^{\alpha}$ and $\bar{p}^{\alpha}$ two non-parallel vectors which ly in resp. on opposite light cones, say for instance $\bar{p}^{\alpha}$ and $\hat{p}^{\alpha}\left(\bar{p}^{\varepsilon} \hat{p}_{\varepsilon}<0\right)$ It is always possible to 
combine $\bar{p}^{\alpha}$ and $\hat{p}^{\alpha}$ with positive factors $a>0, b>0$ to a space-like vector $^{6}$

$$
r^{\alpha}:=a \bar{p}^{\alpha}+b \hat{p}^{\alpha}, \quad r^{\varepsilon} r_{\varepsilon}<0,
$$

which may be written in view of the general definition of all $p^{\alpha}(u)$

$$
r^{\alpha}=T^{\alpha \beta} U_{\beta}, \quad U_{\beta}:=a \bar{u}_{\beta}+b \hat{u}_{\beta}
$$

with $U^{\varepsilon} U_{\varepsilon}>0$. Therefore the 4-velocity $\left(U^{\varepsilon} U_{\varepsilon}\right)^{-1} U^{\alpha}$ implies the spacelike energy flow $\left(U^{\varepsilon} U_{\varepsilon}\right)^{-1} r^{\alpha}$ in contradiction to (3.1). We thus have $p^{\alpha}(u) \neq 0$ for all $u^{\alpha}$. If some $p^{\alpha}(u)$ would be future- and other past-pointing, we could choose because of B) two non-parallel $p^{\alpha}(u)$ which ly in resp. on different light cones. This represents a contradiction to (3.1), as shown above, and completes the proof of (3.2).

\section{Decomposition of $T^{\alpha \beta}$}

We restrict ourselves to Weyl solutions with an energy-momentum tensor $T^{\alpha \beta}$ satisfying the causality condition (3.1) (causal Weyl solution). In order to decompose $T^{\alpha \beta}$ according to (1.1), we adapt a null frame to $j^{\alpha}$. To avoid restrictions we first introduce a new spinor $\varkappa_{A}$ which is proportional to $\varphi_{A}$

$$
\varphi_{A}=\Phi \varkappa_{A}, \quad \Phi \text { complex },
$$

and complete it by $\mu_{A}$ to a basis in spin space:

$$
\varkappa_{A} \mu^{A}=+1, \quad \varepsilon_{A B}=\varkappa_{A} \mu_{B}-\mu_{A} \varkappa_{B} .
$$

Then the vectors $k^{\alpha}, n^{\alpha}, m^{\alpha}, \bar{m}^{\alpha}$ of the corresponding null frame are given by

$$
k^{\alpha} \leftrightarrow \chi_{A} \chi_{\dot{B}}, \quad n^{\alpha} \leftrightarrow \mu_{A} \mu_{\dot{B}}, \quad m^{\alpha} \leftrightarrow \chi_{A} \mu_{\dot{B}}, \quad \bar{m}^{\alpha} \leftrightarrow \chi_{\dot{A}} \mu_{B} . \quad(4.3 \mathrm{a}-\mathrm{d})
$$

Their scalar products vanish with the exception of

$$
k_{\varepsilon} n^{\varepsilon}=+1, \quad m^{\varepsilon} \bar{m}_{\varepsilon}=-1 .
$$

${ }^{6}$ In order to prove (3.11), for example if $\bar{p}^{\alpha}$ and $\hat{p}^{\alpha}$ are both time-like, we take $\left(\bar{p}:=\left(\bar{p}^{\varepsilon} \bar{p}_{\varepsilon}\right)^{\frac{1}{2}}\right.$, $\left.\hat{p}:=\left(\hat{p}^{\varepsilon} \hat{p}_{\varepsilon}\right)^{\frac{1}{2}}\right)$

$$
r^{\alpha}=\frac{\bar{p}}{-\hat{p}^{\varepsilon} \bar{p}_{\varepsilon}} \hat{p}^{\alpha}+\frac{1}{\bar{p}} \bar{p}^{\alpha}, \quad \hat{p}^{\varepsilon} \bar{p}_{\varepsilon}<0 .
$$

Then the following consequence of the signature of the metric

$$
\bar{p}^{2}=\left(\bar{p}^{\varepsilon} \frac{\hat{p}_{\varepsilon}}{\hat{p}}\right)^{2}-\delta^{2}, \quad \delta^{2}>0
$$

leads to $(3.11 \mathrm{~b})$. The other cases can be easily treated in a similar way. 
A null frame $k_{\alpha}^{\prime}, n_{\alpha}^{\prime}, m_{\alpha}^{\prime}, \bar{m}_{\alpha}^{\prime}$ is parallelly propagated ${ }^{7}$ along the $j^{\alpha}$-congruence if

$$
\left(k_{\alpha}^{\prime}\right)^{\circ}:=k_{\alpha \| \varepsilon}^{\prime} k^{\prime \varepsilon}=0, \quad\left(n_{\alpha}^{\prime}\right)^{\circ}=0, \quad\left(m_{\alpha}^{\prime}\right)^{\prime}=0 \quad(4.5 \mathrm{a}-\mathrm{c})
$$

and therefore

$$
\left(\varkappa_{A}^{\prime}\right)^{\circ}:=x_{A \| B \dot{C}}^{\prime} x^{, B} \varkappa^{, \dot{C}}=0, \quad\left(\mu_{A}^{\prime}\right)^{\circ}=0 .
$$

The most general transformation of $\varkappa_{A}$ and $\mu_{A}$ which preserves (4.1) and (4.2) may be written

$$
\varkappa_{A} \rightarrow \varkappa_{A}^{*}:=R^{\frac{1}{2}} e^{\frac{i S}{2}} \varkappa_{A}, \quad \mu_{A} \rightarrow \stackrel{*}{\mu}_{A}:=R^{-\frac{1}{2}} e^{-\frac{i S}{2}}\left(\mu_{A}-R T \varkappa_{A}\right),
$$

with arbitrary $R(x)>0, S(x)$ real and $T(x)$ complex. The corresponding null rotation $k_{\alpha} \rightarrow k_{\alpha}^{*}, n_{\alpha} \rightarrow n_{\alpha}^{*}, m_{\alpha} \rightarrow \tilde{m}_{\alpha}^{*}, \bar{m}_{\alpha} \rightarrow \tilde{m}_{\alpha}$ is:

$$
\begin{aligned}
& k_{\alpha}=R^{-1} k_{\alpha}^{*},
\end{aligned}
$$

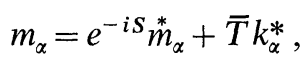

$$
\begin{aligned}
& n_{\alpha}=R{\stackrel{*}{n_{\alpha}}}+R T e^{-i S_{m_{\alpha}}^{*}}+R \bar{T} e^{i S \bar{m}_{\alpha}}+R T \bar{T} k_{\alpha}^{*} .
\end{aligned}
$$

Because of the energy property (3.2), a theorem of Wainwright [4] can be immediately applied to causal Weyl fields leading with (4.1) and (4.3) to a very simple

Decomposition of $T^{\alpha \beta}:$ For all causal Weyl solutions the $j^{\alpha}$-congruence is geodesic and shearfree $(\sigma=0)$ and there exists a special (!) null frame (determined up to scale $R$ and phase $S$ in (4.5)) with respect to which the corresponding $T^{\alpha \beta}$ takes the form

$$
T^{\alpha \beta}=A k^{\alpha} k^{\beta}-4 \Phi \bar{\Phi} \omega\left[m^{(\alpha} \bar{m}^{\beta)}+k^{(\alpha} n^{\beta)}\right],
$$

with

$$
\begin{aligned}
& A:=4 \operatorname{Im}\left(\bar{\Phi} \Phi_{\mid \varepsilon} n^{\varepsilon}+\Phi \bar{\Phi} \gamma\right), \quad \omega:=\operatorname{Im} \varrho, \\
& \varrho:=\varkappa_{A \| B \dot{C}} x^{A} \mu^{B} \chi^{\dot{C}}, \quad \gamma:=\varkappa_{A \| B \dot{C}} \mu^{A} \mu^{B} \mu^{\dot{C}} .
\end{aligned}
$$

According to (4.4) the decomposition (4.9) with (4.10) and (4.11) is characterised by

$$
T^{\alpha \beta} k_{\alpha} k_{\beta}=0, \quad T^{\alpha \beta} n_{\alpha} m_{\beta}=0, \quad T^{\alpha \beta} m_{\alpha} m_{\beta}=0, \quad(4.12 \mathrm{a}-\mathrm{c})
$$

which shows by (4.8) (as mentioned in the theorem) that the special null frame leading to (4.9) is only determined up to an $R$-S -transformation $(T=0)$. That is, (4.9), (4.10) and (4.11) are especially valid for a null frame constructed after (4.3) out of a parallelly propagated $x_{A}^{\prime}$ with (4.6a) and the corresponding $\dot{\mu}_{A}$

$$
x_{A}^{\prime} \mu^{*}=1 \text {. }
$$

7 Parallel propagation is indicated by a dash.

22 Commun. math. Phys., Vol. 21 
Hereby $\Phi$ must be replaced by $\Phi^{\prime}$ with

$$
\Phi^{\prime}:=\varphi_{A} \stackrel{*}{A}^{A}, \quad \varphi_{A}=\Phi^{\prime} \varkappa_{A}^{\prime} .
$$

Accordingly $k^{\prime \alpha} \leftrightarrow x_{A}^{\prime} x_{\dot{B}}^{\prime}$ is a tangent vector of the $j^{\alpha}$-congruence which corresponds to a parametrisation with an affine parameter $r$

$$
x_{A}^{\prime} x_{\dot{B}}^{\prime} \leftrightarrow k^{\alpha}=\frac{\partial x^{\alpha}}{\partial r} .
$$

In referring to this frame, $\omega$ in (4.9) obtains the physical meaning of the twist of the $j^{\alpha}$-streamlines in the sense of ray-optics (comp. $[7,20]$ ).

\section{Asymptotic Smoothness of $\varphi_{A}$}

We obtain the asymptotic behaviour of the components $\tau_{n}$ of $T^{\alpha \beta}$ in (1.1) as a direct consequence of the asymptotic properties of $\varphi_{A}$ without any further explicit requestions concerning metrical quantities. For this purpose we assume

Asymptotic smoothness ${ }^{8}: \varphi_{A}$ with

$$
\varphi_{A}=\Phi^{\prime}(r) x_{A}^{\prime}, \quad\left(x_{A}^{\prime}\right)^{\circ}=0
$$

$\left(r\right.$ parametrizing affinely the corresponding geodesic $j^{\alpha}$-congruence according to (4.15)) is asymptotically smooth, if for $r \rightarrow \infty^{9}$

$$
\Phi^{\prime}=O\left(r^{-N}\right) \quad \text { with } \quad N \geqq 0
$$

and further the derivations of $\Phi^{\prime}(r)$ with respect to any along the $j^{\alpha}$ congruence parallelly propagated null frame (4.3) satisfy:

$$
\begin{aligned}
\left(\Phi^{\prime}\right)^{\cdot}: & =\Phi_{\mid \varepsilon}^{\prime} k^{{ }^{\varepsilon}}=O\left(r^{-N-1}\right), \\
\Phi_{\mid \varepsilon}^{\prime} n^{\prime} & =O\left(r^{-N}\right) .
\end{aligned}
$$

It follows from (4.6) and (4.7b) that every function $T$ which establishes the connexion between two parallelly propagated null frames, is of the order one in $r$. Therefore in consequence of $(5.2 b)$ and (5.2c) we have in view of $(4.8 \mathrm{~b}, \mathrm{c})$ for all these null frames.

$$
\Phi_{\mid \varepsilon}^{\prime} \operatorname{Re} m^{\prime \varepsilon}=O\left(r^{-N}\right), \quad \Phi_{\mid \varepsilon}^{\prime} \operatorname{Re} \bar{m}^{\varepsilon}=O\left(r^{-N}\right) .
$$

${ }^{8}$ Compare the corresponding definitions in [12, 21] and especially in [9].

9 Landau's order symbols represent statements about limiting processes: $A(r, x)=O(B(r, x))$ for $r \rightarrow \infty$ is defined by

$$
\lim _{r \rightarrow \infty}\left|\frac{A}{B}\right|<d(x) \quad \text { with } \quad \frac{\partial d}{\partial r}=0 .
$$


To obtain a physical interpretation of the requestion (5.2), we introduce an observer $u^{\text {' }}$ with

$$
\left(u^{\prime \alpha}\right)^{\cdot}:=u^{\prime \alpha}{ }_{\| \varepsilon} k^{\ell \varepsilon}=0,
$$

and a special parallelly propagated null frame which enables the following decomposition of $u^{\alpha}$

$$
u^{\prime \alpha}=\frac{1}{2 u^{\prime \varepsilon} k_{\varepsilon}^{\prime}} k^{\prime \alpha}+\left(u^{\gamma^{\varepsilon}} k_{\varepsilon}^{\prime}\right) n^{\prime \alpha}, \quad\left(n^{\prime \alpha}\right)^{\cdot}=0
$$

This taken together with (2.8a) shows that $\left|\Phi^{\prime}\right|^{2}$ (neglecting a factor $u^{\prime} k_{\varepsilon}$, which is independent of $r$ and can be removed by changing the affine parameter) represents the probability density $W\left(u^{\prime}\right)$ :

$$
\left|\Phi^{\prime}\right|^{2}=\frac{W\left(u^{\prime}\right)}{u^{\prime} k_{\varepsilon}^{\prime}}, \quad\left(u^{\prime} k_{\varepsilon}^{\prime}\right)^{\cdot}=0 .
$$

Therefore by (5.2a) incoming radiation is excluded. The analogous interpretations of (5.2b) and (5.2c) are less physically intuitive.

\section{Asymptotic Behaviour}

In order to determine the asymptotic behaviour of the remaining optical parameters $\Theta$ (expansion) and $\omega$ (twist), we contract the Weyl equation (2.2b) with $x^{\prime \dot{C}}$ and obtain with (4.2b), (4.11a) and (4.14b) because of $(4.6 a)$ :

$$
\left(\Phi^{\prime}\right)^{\cdot}=\varrho \Phi^{\prime}, \quad \varrho:=-(\Theta+i \omega) .
$$

Then the asymptotic smoothness $(5.2 \mathrm{~b})$ leads to

which implies

$$
\varrho=O\left(r^{-1}\right),
$$

$$
\omega=O\left(r^{-1}\right), \quad \Theta=O\left(r^{-1}\right) .
$$

$(6.3 \mathrm{~b})$ indicates once more outgoing radiation.

We take advantage of the causality condition (3.1) in starting from the special form (4.6) of $T^{\alpha \beta}$ obtained with respect to $\varkappa_{A}^{\prime}, \tilde{\mu}_{A}$ and $\Phi^{\prime}$ of (4.13) and (4.14). However, the asymptotic behaviour is defined with respect to a parallelly propagated null frame $k_{\alpha}^{\prime}=k_{\alpha}, n_{\alpha}^{\prime}, m_{\alpha}^{\prime}, \bar{m}_{\alpha}^{\prime}$ with (4.6). Therefore we perform in (4.9) a null rotation (4.8) with

and obtain

$$
R=1, \quad S=1, \quad T=\ddot{\mu}_{A}^{*} \mu^{A}
$$

$$
\begin{aligned}
T_{\alpha \beta}= & \tau_{1} k_{\alpha}^{\prime} k_{\beta}^{\prime}+\tau_{2} k_{(\alpha}^{\prime} n_{\beta)}^{\prime}+\tau_{4} m_{(\alpha}^{\prime} \bar{m}_{\beta)}^{\prime} \\
& +\operatorname{Re}\left(\tau_{5} k_{(\alpha}^{\prime} m_{\beta)}^{\prime}\right)
\end{aligned}
$$


with

$\tau_{1}=4 \operatorname{Im}\left(F_{\varepsilon} n^{\prime \varepsilon}+2 F_{\varepsilon} \operatorname{Re}\left(m^{\prime}{ }^{\varepsilon} T\right)+F_{\varepsilon} k^{\prime}\left|T^{2}\right|+\Phi^{\prime} \bar{\Phi}^{\prime} \gamma\right)-8 \Phi^{\prime} \bar{\Phi}^{\prime} \omega|T|^{2}$, (6.6a)

$\tau_{2}=\tau_{4}=-4 \Phi^{\prime} \bar{\Phi}^{\prime} \omega, \quad \tau_{5}=-16 \Phi^{\prime} \bar{\Phi}^{\prime} \omega T$

and $F_{\varepsilon}$ defined by

$$
F_{\varepsilon}:=\bar{\Phi}^{\prime} \Phi_{\mid \varepsilon}^{\prime} .
$$

The orders of the $\tau_{n}$ for $r \rightarrow \infty$ follow with (5.2), (5.3) and (6.3a):

$$
\begin{aligned}
& \tau_{1}=O\left(r^{-2 N}\right)+O\left(r^{-2 N}|T|\right)+O\left(r^{-2 N-1}|T|^{2}\right)+O\left(r^{-2 N} \gamma\right), \\
& \tau_{2}=\tau_{4}=O\left(r^{-2 N-1}\right), \quad \tau_{5}=O\left(r^{-2 N-1} T\right) .
\end{aligned}
$$

To give $\tau_{1}$ a uniform order, we introduce

$$
E(r, x):=\max \left\{\begin{array}{l}
r^{-2 N} \\
r^{-2 N}|T| \\
r^{-2 N-1} T^{2} \\
r^{-2 N} \cdot \gamma
\end{array}\right.
$$

By $(6.8) E(r, x)$ is defined pointwise as the maximum value of the functions and $(6.7 \mathrm{a})$ takes the form

$$
\tau_{1}=O(E)
$$

Finally a straightforward calculation (distinguish e.g. the cases $T=1,|T|<1$ and $|T|>1$ ) leads for $r>1$ independently of $T(x)$ to

$$
\begin{aligned}
& E \geqq r\left(r^{-2 N-1}|T|\right), \\
& E \geqq r\left(r^{-2 N-1}\right),
\end{aligned}
$$

which means that the order of the radiation term $\tau_{1}$ in (6.5) exceeds asymptotically for $r \rightarrow \infty$ the orders of the other terms at least by a factor $r$.

Asymptotic behaviour ${ }^{10}$ : If a solution $\varphi_{A}$ of the Weyl equation in some (!) Riemann space is

a) causal (in the sense of Section 3)

$\beta$ ) asymptotically smooth (in the sense of Section 5)

we may parametrize the corresponding geodesic $j^{\alpha}$-congruence affinely with $r$, and decompose its energy-momentum tensor $T^{\alpha \beta}$ according to (1.1)

${ }^{10}$ Following the lines of our calculation, it can be easily shown that the same behaviour is also valid for asymptotically smooth solutions $\varphi_{A}$ of the coupled Weyl-Einstein system (2.1) and (2.2) (or for a vacuum metric), which only satisfy $T^{\alpha \beta} u_{\alpha} u_{\beta} \neq 0$ for all $u^{\alpha}$ instead of (3.2), and furthermore $\ddot{\Phi}=O\left(r^{-N-2}\right)$ in addition to (5.2).

In particular, with the Sachs equation $[20] \dot{\varrho}=\varrho^{2}+\sigma \bar{\sigma}+R_{\alpha \beta} j^{\alpha} j^{\beta}$ which describes the propagation of the optical scalars of null geodesics, one obtains $\sigma=O\left(r^{-1}\right)$. This enables the treatment of the new term $4 \operatorname{Im}\left(\bar{\Phi}^{\prime} \Phi^{\prime} \sigma T^{2}\right) k_{\alpha}^{\prime} k_{\beta}^{\prime}+\operatorname{Re}\left(i \bar{\Phi}^{\prime} \Phi^{\prime} \bar{\sigma} m_{\alpha}^{\prime} m_{\beta}^{\prime}\right)+8 \operatorname{Re}\left(i \bar{\Phi}^{\prime} \Phi^{\prime} \bar{\sigma} \bar{T} k_{(\alpha}^{\prime} m_{\beta)}^{\prime}\right)$ in the corresponding decomposition (comp. [4]) of $T^{\alpha \beta}$. 
with respect to a null frame $k^{\alpha}=\frac{\partial x^{\alpha}}{\partial r} \sim j^{\alpha}, n^{\alpha}, m^{\alpha}, \bar{m}^{\alpha}$ which is parallely propagated along the $j^{\alpha}$-congruence. Then asymptotically for $r \rightarrow \infty$, the order of the pure radiation term $\tau_{1}=T^{\alpha \beta} n_{\alpha} n_{\beta}$ predominates over the orders of the other terms $\tau_{i}$ at least by a factor $r$ (radiation behaviour).

The author wishes to thank Prof. H. Dehnen for stimulating discussions.

\section{References}

1. Griffiths, J. B., Newing, R. A.: J. Phys. A: Gen. Phys. 3, 136 (1970).

2. - - J. Phys. A: Gen. Phys. 3, 269 (1970).

3. - - Preprint. University College of North Wales, Bangor, Great Britain: 1970 Submitted to J. Phys. A: Gen. Phys.

4. Wainwright, J.: Preprint. University of Waterloo, Waterloo, Canada: 1970. Submitted to J. Math. Phys.

5. Trim, D.: Preprint. University of Waterloo, Waterloo, Canada 1970.

6. Audretsch, J., Graf, W.: Commun. math. Phys. 19, 315 (1970).

7. Pirani,F.A.E.: In: Gravitation: an introduction to current research. L. Witten (ed.). New York-London: J. Wiley 1962.

8. Plebanski, J.: Acta Phys. Polon. 26, 963 (1964).

9. Penrose, R.: Proc. Roy. Soc. London A 284, 159 (1965).

10. Bondi, H., van den Burg, M.G. J., Metzner, A. W.: Proc. Roy. Soc. London A 269, 21 (1962).

11. Sachs, R. K.: Proc. Roy. Soc. London A 270, 103 (1962).

12. Newman, E., Penrose, R.: J. Math. Phys. 3, 566 (1962).

13. Kozarzewski, B.: Acta Phys. Polon. 27, 775 (1965).

14. Jordan, P., Ehlers, J., Sachs, R. K.: Akad. Wiss. Lit. Mainz Abh. Math.-Nat. K1. Nr. 1 (1961).

15. Schmutzer,E.: Relativistische Physik (Klassische Theorie). Leipzig: B. G. Teubner 1968.

16. Brill,D. R., Wheeler, J.A.: Rev. Mod. Phys. 29, 465 (1957).

17. Pirani, F.A.E.: In: Bondi, Pirani, Trautmann: Lectures on general relativity. Englewood Cliffs, N. J.: Prentice Hall 1964.

18. Penrose, R.: Ann. Phys. 10, 171 (1960).

19. - In: Batelle Rencontres. C. M. DeWitt, J. A. Wheeler (ed.). New York-Amsterdam: W. A. Benjamin 1968.

20. Sachs, R. K.: Proc. Roy. Soc. London A 264, 309 (1961).

21. - Phys. Rev. 128, 2851 (1962).

J. Audretsch

Fachbereich Physik der Universität

Postfach 733

BRD-7750 Konstanz, Germany 